\title{
e-Tools: An agent coordination layer to support the mobility of persons with disabilities.
}

\author{
Cristian Barrué ${ }^{1}$, Ulises Cortés ${ }^{1}$, Antonio B. Martínez ${ }^{1}$, Josep Escoda ${ }^{1}$, \\ Roberta Annicchiarico ${ }^{2}$, and Carlo Caltagirone ${ }^{2,3}$ \\ 1 Universitat Politècnica de Catalunya. cbarrue, ia@lsi.upc.edu \\ 2 IRCCS Fondazione Santa Lucia. \\ r.annicchiarico,c.caltagirone@hsantalucia.it \\ 3 Università di Roma "Tor Vergata"
}

\begin{abstract}
This paper outlines the development and integration of an agent coordination layer with a robotic platform to support senior citizens or persons with disabilities. This platform is situated in a given context (such as a Hospital) and it is intended to enhance user's mobility and autonomy. This objective is performed in a safe and sound fashion that meets the sets of laws, norms or protocols which rule the selected context.
\end{abstract}

\section{Introduction}

Disability is usually defined as the degree of difficulty or inability to independently perform basic Activities of Daily Living (ADLs) or other tasks essential for independent living, without assistance. It is generally recognized, however, that disability is not merely a function of underlying pathology and impairment, but involves an adaptive process, which is subject to a host of individual (psychosocial) and ecologic (environmental) factors. Currently we define this complex syndrome as Functional Disability $(F D)[1]$. In fact, $F D$ has to be intended as the result of the interaction of different individual components of compromised functions: physical, emotional, and cognitive aspects usually interact to produce a comprehensive disability which is more than the simple addition of the single impairments, affecting the patient's global function and his self-dependency [2].

Subjects with $F D$ and affected by chronic diseases or outcomes of acute events, such as Parkinson disease, dementia, stroke, accidents, etc. represent a heterogeneous category of individuals: each user may be affected by at least one of these symptoms: ambulatory impairment, memory loss, staggering gait, ataxia, visio-spatial dysfunction, aphasia. In other words, each and every one of these features can be combined differently and with different severity in individual users, impairing their self-dependency and worsening their quality of life. To solve a complex syndrome as FD a number of approaches have been proposed, the most comprehensive being represented by the rehabilitative team. In recent years the introduction of new technologies has been proposed. Assistive

Please use the following format when citing this chapter:

Barrué, C., Cortés, U., Martínez, A.B., Escoda, J., Annicchiarico, R., Caltagirone, C., 2006, in IFIP International Federation for Information Processing, Volume 217, Artificial Intelligence in Theory and Practice, ed. M. Bramer, (Boston: Springer), pp. $425-434$. 
technologies (AT) may be defined as devices and techniques that can eliminate, ameliorate, or compensate for functional limitations. They help people with disabling conditions interact more efficiently and effectively with their social and physical environment [3].

The growing attention given to these citizens creates a need for deploying new types of services to sustain independence and preserve quality of life. Many of those services need to have access to personal data, e.g., user's clinical data or the continuous tracking of that person in a given environment (inside a hospital or a house). These services not only need to be efficient but must also comply with the laws and norms which apply in a country or a region as well as the protocols that rule in a hospital and user or condition specific rules.

One of the most featured tools used by our target population is the wheelchair; unfortunately it is one of the most difficult devices to be autonomously used (requiring control, physical interaction and also planning/ strategy for navigation or obstacle avoidance). One possible solution is represented by the use of power wheelchairs, but the extreme difficulty persons with severe disabilities are taught to manoeuvre them is an example of difficult interaction with AT: nearly one half of the users unable to control a power wheelchair by conventional methods would benefit from an automated navigation system. This indicates a need, not only for more innovation in steering interfaces, but for entirely new technologies for supervised autonomous navigation [4].

In this paper, we present an assistive device realized for a person with disabilities who - due to different pathologies - is no longer able to independently provide to his own self-care [5], and who needs the support of a second person to perform even the simplest every-day activities, referred to as ADLs. The aim is the integration of agent technology with other technologies to build specific $e$-Tools for the target group. $e$-Tools stands for Embedded Tools, as we aim to embed intelligent assistive devices in hospitals, homes and other facilities, creating ambient intelligence environments to give support to users and caregivers. However, according to the type of pathology, the impairment of physical and/or cognitive abilities restricts the possibility of precisely defining the control or maintaining it over a long period of time. Following these remarks, the intelligent platform can provide assistance by suggesting the preferred direction (for example, direction that the user is used to take) so that the user does not have to redefine the control during motion.

In $\S 2$ we describe the $e$-Tools project and its current architecture. In $\S 3$ we explain the approach followed to design the agent coordination layer to coordinate interaction between the software agents, the robotic platform(s) and the environment, and in $\S 4$ we present some conclusions.

\section{2 e-Tools PROJECT}

In order to provide proper healthcare management (embedded monitoring and diagnosis functionalities) and to ease the relation of users with other people 
and the environment we propose to build an integrated system in which the environment (a home, a hospital) and the people inside it (users, carers) are connected. This approach integrates Ambient Intelligence (sensors, automatic dialers, automatic cooling and heating system) with solutions related to MultiAgent Systems (MAS), machine learning and other AI techniques, affective computing, wireless devices and robotics. The typical environment considered is a hospital for the neuro-motor rehabilitation, referring to a real institution represented by IRCCS S. Lucia Foundation, located in Rome.

Our target population is characterized by different profiles of $F D$. That means that an electric-powered wheelchair should be flexible to the needs of different users; at the same time, the wheelchair has to be flexible to the needs of the same user in different times: users go a pathway of changing (dys) functionality - possibly improving - during their illness. The robotic platform we propose (see figure $2 \mathrm{~b}$ ) can be used to support the mobility of senior citizens or persons facing a disability that have a standard wheelchair [5]. That is the platform will be able to drive any standard wheelchair and to provide services through its interface. In this case, wheelchairs will be driven, in an indoor environment, by the robotic platform, supporting the mobility of the person. The platform has to show complete autonomy in tasks such as path planning and location in the environment, and at the same time pay attention to the user's needs and requests. Although the robotic platform will be functioning in a well-known environment, structural elements like corridors, rooms, or halls may differ. The autonomous platform for persons with $F D$ can be considered as an intelligent vehicle whose main feature is moving in areas that are time-dependent and wellknown but changing. When a person with disabilities moves in such a platform, he must have the impression that he is in control of the vehicle at all times.

In order to achieve such complex and adaptive behaviour, the system will combine the wheelchair hardware of the robotic platform with a MAS that controls and adapts the behavior of the chair, monitors the state of the user and interacts with him/her through a flexible interface that provides more or less assistance in navigation, depending on the user's individual capabilities. Navigation should be autonomously controlled by the MAS most of the times, to relieve the user from tedious low-level decision-making tasks. To make this possible, the platform will be wirelessly connected to the environment, where an agent-based coordination layer will provide extra information to the robotic platform MAS. To support the agent-based coordination layer and to connect it with the robotic platform MAS, active landmarks will be placed. These active landmarks are small wireless machines installed in some strategic places of an area to transmit local information to the mobile entity. In order to filter all the information received from the sensors and send only relevant information to a given platform, each room must be monitored and controlled by a MAS. This agent-based controller can proactively make decisions about room conditioning, or process sensor signals in order to extract meaningful information (e.g. to track a given person in the room). 
These elements can be structured into the architecture (see figure 1a) as it was introduced in [3].
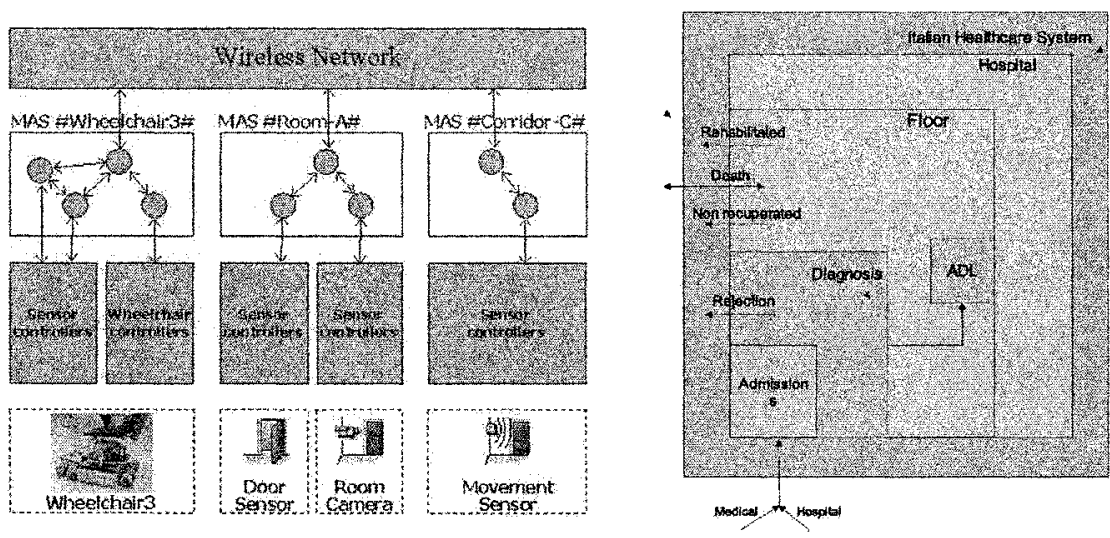

Fig. 1. a) (left) the system levels, b) (right) IRCCS Sta. Lucia organizational simplified model

\section{Designing the $e$-Tools Coordination Layer}

To deploy $e$-Tools in a real, complex environment such as the IRCCS Santa Lucia, the design and implementation of the agents in the agent-based coordination layer should be done taking into account not only the organizational structures and internal regulations of the IRCCS itself, but also any external requirement defined by the context of IRCCS. In order to introduce all these factors in the design of the multi-agent architecture, we will use the HARMONIA approach, introduced in [6]. The idea is to define the agent-based coordination layer as an electronic institution, where not only coordination between the users, the medical staff and the $e$-Tools is provided but also safety mechanisms are included to ensure that the behaviour of the system as a whole and of each individual agent is both legal and acceptable from the institutional perspective. The institutional model and the concrete role definition was introduced in [7].

The aim of assistive MAS is to provide a series of services to complement and enhance user's autonomy, in many cases severely constrained by their own $F D$, and to give support in rehabilitation tasks. In the other hand the MAS will be collecting information for the medical users like medical data, behavioural data, driving performance, that will be used in medical studies to support diagnosis and treatment. 


\subsection{The Procedure Level: IRCSS agent-based coordination layer}

The Procedure Level focus on the implementation of the Virtual IRCSS by means of an agent-based coordination layer. At this level the main activity is the definition of the agents that will (a) enact the roles defined in the Concrete Level and (b) meet the norms and rules defined in the Concrete Level.

Our MAS has the following basic agents. First, we have a Patient Agent $(p a)$, for each client, that could be integrated into the platform or connected to it, for example, using a PDA. This agent enacts the Patient role and, therefore, it should provide all the available and permitted services to the user and it should take care of his/hers personal security. Once in a floor, each accepted patient $p_{i}$ is provided with a personal agent $p a_{i}$ that allows her to use all the available and authorized agent-based services. Each $p a_{i}$ is personalized to its owner so its identity is linked to her and makes it unique. Each pa provides a personalized way of interaction with the user and therefore users could use it to ask for help or to ask the platform to drive her/him to a given place into the permitted space or to ask the system to show a possible path to the destination. It should ensure that the user is aware of the activities he (the user) is expected to perform, the pa should augment the likelihood that he will perform at least the compulsory activities such as taking medicines). An important task is however to prevent the user being overly reliant on the system. Also, the pa takes responsibility for auditing the user's biometric signals and acting as a consequence.

The Medical Agents ( $m a$ ) enact the Caregiver role, or, (to be more precise), one on the subroles of Caregiver, depending on the staff member it is representing and its position in the organizational structure of IRCSS. The $m a$ will be situated in the PCs belonging to the caregivers as well as in their individual PDA. The $m a$ is in charge of managing all the user's request messages. It also serves all caregivers' requests for user information (e.g., a caregiver could use his Medical Agent ( $m a$ ) to ask for: Tell me Patient,'s (corporal) Temperature or Give me the Patient, 's (actual) Status or Tell me where Patient ${ }_{i}$ 's is). Also, the $m a$ notifies the caregiver of any anomaly in the user's biometric signals and it will generate a request for help, if needed. A range of emergency situations can be avoided with such systematic data collection (e.g. user's catastrophic reactions). A special task to be performed is to initialize the daily activities for each user, as well as any constraints on, or preferences regarding, the time or manner of their performance. This schedule may then be modified in several ways: (a) the user or a caregiver may add new activities (e.g. add a new session at the gym), (b) the user or a caregiver may modify or delete activities already in the schedule, (c) the user performs one of the scheduled activities; or (d) as time goes the schedule is automatically up-dated (e.g. changes in priorities).

The Sensor Network Agent (sna) undertakes responsibility for the network of sensors. It enacts the Environment Sensors Manager role, and its basic target is to distribute the information from all available sensors to all the agents that maybe interested and avoiding to send irrelevant information. Also, it has 
to report problems and failures in the network. The list of current sensors for this space include: movement, landmarks, cameras, presence, etc.

Finally, the Main Agent $(M A A)$ enacts the Coordinator and Information Manager roles. Therefore, the $M A A$ should keep the coherence of all clients' schedules. $M A A$ is responsible for the protection of all the clients' personal data (this fact is expressed as an Obligation, for example in equation 3 ). If needed for scalability reasons, the Coordinator role can subsequently be distributed in several $M A A$ 's which should coordinate among themselves in order to ensure that clinical standards can always be met with certainty. All these agents share an ontology that allow them to exchange information for carrying out their activities. This ontology contains the description of the elements of the physical environment as well as those of the conceptual world that the agents need to know. Also, it contains the actions and propositions that give support to the communicative acts that put them in contact.

\subsection{Study}

In a controlled experiment carried out at the IRCCS outdoor facilities we got some real users to use a prototype of the MAS controlled platform. This MAS was equiped with prototypes of pa $m a$ and the sna that allowed users to choose a path to be followed autonomously by the platform in the garden.

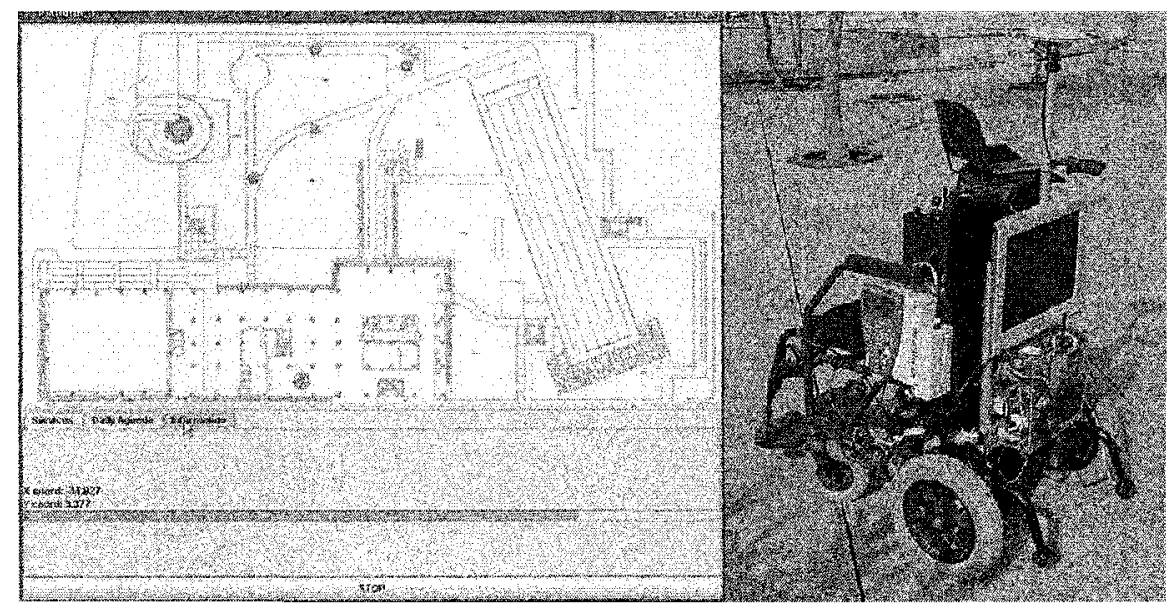

Fig. 2. a) (left) a screen capture of the pa monitoring a plan execution, b) (right) The outdoors robotic wheelchair

Figure 2 shows a $p a$ capturing the route that the platform is executing on the user's request. It shows the map of the garden indicating the updated platform's position. 
Another test was designed to enhance user's autonomy and to evaluate their response to the shared control. For this a specific path was designed and marked on the ground so the users could follow it. An error threshold was established in $30 \mathrm{cms}$ from the center line of the given path, when the user drove the platform away of the threshold the MAS got the control returning the platform to the center of the path with the proper orientation. After that, the MAS returned the manual control to the user.

\subsection{Virtual Hospital}

In figure $1 b$ we show a simplified model of IRCCS Sta Lucia's actual organization and explain some of the scenes that occurs in a hospital to show HARMON $I A$ 's power to model the agent-mediated interaction in an e-organization.

Patient's are derived into the IRCCS Sta Lucia either by their doctor or from a Hospital to be rehabilitated - to rehabilitate patients from neurological accidents is one of the Sta Lucia's main objectives - there a diagnosticated and either sent to a floor or rejected. Once in a floor, each accepted patient is provided with a personal agent $p a_{i}$ that allows her to use all the available and authorized agent-based services. Each $p a_{i}$ is personalized to its owner so its identity is binded to her and makes it unique.

In the following, we will describe typical scenes where $p a_{i}$ interacts with other agents to accomplish some generic tasks.

Requesting a Plan Here we will illustrate the treatment given to the creation of a plan for a given agent $p a_{i}$ willing to go from her actual position to a new one $X$. In our scenario there are two different ways to start this process. Either a user $p_{i}$ requests the system for a plan to go to $X$-using her $p a_{i}$-or the agent itself initiates the request. We assume that if the $p a_{i}$ or $a_{i}$ wants to go to $X$ has the goal to go there $G_{a_{i}}$ go $\left(a_{i}, x\right)$. This goal is state in expression 1 .

$$
\begin{aligned}
G_{a_{i}} \operatorname{go}\left(a_{i}, x\right)= & D_{a_{i}} \operatorname{go}\left(a_{i}, x\right) \wedge \neg B_{a_{i}} i n\left(a_{i}, x\right) \\
& \wedge B_{a_{i}} \text { achieve }\left(i n\left(a_{i}, x\right)\right)
\end{aligned}
$$

Once the request arrives into the system we find that the system $(s l)$ has the obligation to find out if $p a_{i}$ has the necessary permissions to go $X$ before it creates and delivers a plan. Expression 2 depicts all this process.

$$
\begin{aligned}
O_{s l} & \left(\operatorname{check}\left(s l, P_{a_{i}} \operatorname{go}\left(a_{i}, x\right)\right)\right. \\
& \left.<\operatorname{send}\left(s l, a_{i}, \text { Plan }\right)\right)
\end{aligned}
$$

In a hospital or an assisted living facility there are places to which access is forbidden for several reasons to different users. One special case are stairs and lifts. The system should assure that if $p a_{i}$ is not allowed to change floor she will not use the chairs nor the lifts. Also, in the case of a platform they cannot be used to try to reach the stairs. 
Requesting Status A normal action in the daily activity of a healthcare giver is to collect information about a user. In our context this can be achieved using the $e$-Tools facilities. For example, a caregiver could use his Medical Agent

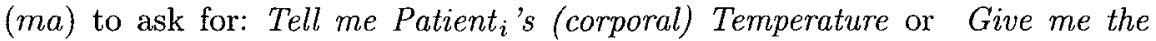
Patient $_{i}$ 's (actual) Status or Tell me where Patient ${ }_{i}$ is? again, before to receive that information the system has the obligation to verify if the requesting $m a_{k}$ has the appropriate permissions and it will write down the transaction in an special audit file, if finally it decides to deliver the data. This is expressed in expression 3 .

$$
\begin{aligned}
O_{s l} & (\text { register }(s l, \log , \\
& \text { personal_data_request(sta.tus, } \left.\left.\left.m a_{k}, a_{i}\right)\right)\right)
\end{aligned}
$$

this is because the system should respect the Law on Personal Data Protection [8].

An exception occurs in the case of an emergency. If the system acknowledge that a $p a_{i}$ is involved in an emergency and this user's clinical history (HC) is requested by a caregiver's agent $m a_{k}$ the system will deliver the information without creating an entrance to the audit files but it will create an obligation in $m a_{k}$ such that after the end of the emergency it should create an entrance to the audit files as expressed in equation 5 . Then, IF Context $=$ Emergency the $O_{s l}$ is created, see expression 4.

$$
\begin{gathered}
O_{s l}\left(\operatorname{inform}\left(s l, \operatorname{access}\left(m a_{k}, \mathrm{HC}\left(p_{i}\right)\right)\right)\right) \\
O_{m a_{k}}\left(\text { declare_access }\left(m a_{k}, \mathrm{HC}\left(p_{i}\right)\right)>\text { Emergency }=\mathrm{NIL}\right)
\end{gathered}
$$

Help Request Manager When a gethelp request arrives into the system a new situation arises. The system starts to play as Help Request Manager. This request could be originated from two different sources: In the first the $a_{i}$ decides that her owner is in problematic situation and it decides to send a request for help (see equation 6). In the second case is the user himself who calls for help (see equation 7).

$$
\begin{aligned}
G_{a_{i}} \text { gethelp }\left(p_{i}, c g_{j}\right) & =D_{a_{i}} \text { gethelp }\left(p_{i}, c g_{j}\right) \quad G_{p_{i}} \text { gethelp }\left(p_{i}, c g_{j}\right) & =D_{p_{i}} \operatorname{gethelp}\left(p_{i}, c g_{j}\right) \\
& \wedge \neg B_{a_{i}} \text { helpattend }\left(c g_{j}, p_{i}\right) & \wedge \neg B_{a_{i}} \text { helpattend }\left(c g_{j}, p_{i}\right) \\
& \wedge B_{a_{i}} \text { needhelp }\left(p_{i}\right) \quad(6) \quad & \wedge B_{a_{i}} \text { needhelp }\left(p_{i}\right)
\end{aligned}
$$

When the system $s l$ gets a request as the ones depicted in equations 6 and 7 it should notify it to all the available $m a_{j}$ and verify that this $p_{i}$ has not pending requests. This behaviour is defined by equation 8

$$
\begin{aligned}
O_{s l}\left(\operatorname{check}\left(\neg \text { wait_attend }_{p_{i}}\right)\right) & <\operatorname{notify}\left(s l, m a_{k}, \text { request }_{p_{i}}\right) \\
& \left.\wedge \text { wait_attend } p_{p_{i}}\right)
\end{aligned}
$$




\section{Study and Conclusions}

An experiment was performed consisting on the pursuit of a simple straight line drawn on the floor using a wheelchair in three different scenarios. The test evaluates the performance of the user navigation using first a conventional wheelchair, secondly a standard electric powered wheelchair manually controlled and, then an autonomous wheelchair prototype with shared control. These tests were all designed to measure the interactions among physical and cognitive capabilities to perform this simple task with more or less assistance. In our experiments, the wheelchairs have been tested by a group of 24 neurological and orthopaedic inpatients who needed a daily use of wheelchair - 10 males $(41.7 \%)$ and 14 females ( $58.3 \%$ ); mean age 67.7 years - during a four-week period. Exclusion criteria were: patients bedridden, patients walking autonomously, presence of global aphasia and blindness. Each subject underwent a structured clinical evaluation and assessment of cognitive, emotional and functional abilities. This entire procedure was performed by a trained physician.

Of all the 24 persons involved in the experiment, 14 finished correctly the first test, while only 12 were able to finish the second. The third test, though, was successfully completed by all 24 persons.

Assistive technologies [9] open a new option to show the versatility and robustness of agent systems on a large scale, reducing the fragility of the conventional software bringing an infrastructure constructed with tolerance to the uncertainty, the inconsistency and different points of view. Technology will never alleviate all problems that aged population and persons with disabilities face, in special those that require a human interaction. $e$-Tools may also alleviate caregivers from routine tasks and could improve also their quality of life diminishing their degree of distress.

Our main effort is to develop $e$-Tools capable to supply different levels of disability and to satisfy the needs of each user through its flexibility. We focused on one of the most common problems assistive devices are adopted for: mobility limitations and their correlates. To date, we have developed a) a fully-functional intelligent robotic platform for wheelchairs [5], along with 2) the software to enable to install the agent layer, and 3) a first prototype of the agent coordination layer, following the role structure and objective division defined in $\S 3$, but still we have to develop the second layer and to integrate the whole architecture at the IRCCS Santa Lucia in Rome.

Our approach proposes a real integration of heterogeneous technologies to serve to disabled and senior citizen in a non-intrusive way and securing the personal information of the users, working towards an integral solution beyond existing efforts that try to solve subsets of problems. The $e$-Tools philosophy puts a lot attention in create tools to help users to recover their autonomy in as much as possible. In this sense, our tools are meant not to override any of the personal capabilities of the user if $\mathrm{s} /$ he can solve a situation on their own. 


\section{References}

1. Guralnik, J., Land, K., Blazer, D., Fillenbaum, G., Branch, L.: Educational status and active life expectancy among older blacks and whites. The New England journal of medicine 329 (1993) 126-127

2. Fehr, L., Langbein, W., Skaar, S.: Adequacy of power wheelchair control interfaces for persons with severe disabilities: A clinical survey. Journal of Rehabilitation Research and Development 37 (2000)

3. Cortés, U., Annicchiarico, R., Vázquez-Salceda, J., Urdiales, C., Cañamero, L., López, M., Sànchez-Marrè, M., Caltagirone, C.: Assistive technologies for the disabled and for the new generation of senior citizens: the e-Tools architecture. AI Communications 16 (2003) 193-207

4. Urdiales, C., Bandera, A., Pérez, E., Poncela, A., Sandoval, F. In: Hierarchical planning in a mobile robot for map learning and navigation. Physica-Verlag, Heidelberg (2002)

5. Martínez, A., Escoda, J., Benedico, T., Cortés, U., Annicchiarico, R., Barrué, C., Caltagirone, C.: Patient driven mobile platform to enhance conventional wheelchair, with mul- tiagent system supervisory control. In: Multi-Agent Systems and Applications IV: 4th International Central and Eastern European Conference on Multi-Agent Systems, CEEMAS 2005. Volume 3690 of Lecture Notes in Computer Science., Springer-Verlag (2005) 92-101

6. Vázquez-Salceda, J.: The Role of Norms and Electronic Institutions in Multi-Agent Systems: The HARMONIA Frameworks. Whitestein Series in Software Agent Technologies (2004)

7. Barrué, C., U.Cortés, Martínez, A., Vázquez-Salceda, J., Annicchiarico, R., C.Caltagirone: An e-institution framework for the deployment of e-tools to support persons with disabilities. (In: $3^{r d}$ Workshop on Agents Applied in Health Care. 19th International Joint Conference on Artificial Intelligence (IJCAI-05)) 6-15

8. : Directive 95/46/CE of the European Parliament and of the Council of 24 october 1995 on the protection of individuals with regard to the processing of personal data and of the free movement of such data (1995)

9. Mittal, V., Yanco, H., Aronis, J., Simpson, R., eds.: Assistive Technology and Artificial Intelligence: Applications in Robotics, User Interfaces and Natural Language Processing. Volume 1458 of Lecture Notes in Artificial Intelligence. Springer-Verlag, Berlin (1998) 